\title{
HEAT AND MASS TRANSFER IN A SECOND GRADE FLUID OVER A STRETCHING VERTICAL SURFACE IN A POROUS MEDIUM
}

\author{
I.G. BAOKU* \\ Department of Physical Sciences \\ Bells University of Technology \\ Ota, NIGERIA \\ E-mail: baokuig@bellsuniversity.edu.ng \\ Y.S. ONIFADE \\ Department of Physics \\ Federal University Petroleum Resources \\ Effurun, Warri, NIGERIA \\ L.O. ADEBAYO \\ Department of Chemical Sciences, Crescent University \\ Abeokuta, NIGERIA \\ K.M. YUSUFF \\ Department of Statistics, University of Ibadan \\ Ibadan, NIGERIA
}

\begin{abstract}
The investigation deals with the combined heat and mass transfer in a mixed convection boundary layer flow over a stretching vertical surface in a porous medium filled with a viscoelastic second grade fluid. The partial differential equations governing the model have been transformed by a similarity transformation and the system of coupled-ordinary differential equations is solved by employing the shooting method with the fifth-order Runge-Kutta-Fehlberg iteration technique. Effects of various values of physical parameters embedded in the flow model on the dimensionless velocity, temperature and concentration distributions are discussed and shown with the aid of graphs. Numerical values of physical quantities, such as the local skin-coefficient, local Nusselt number and local Sherwood number are presented in a tabular form. It is observed that the boundary layer fluid velocity increases as the second grade parameter, mixed convection parameter and Prandtl number increase.
\end{abstract}

Key words: heat and mass transfer, second grade fluid, porous medium, stretching surface.

\section{Introduction}

The phenomenon of heat and mass transfer has been the object of extensive research due to its applications in science and technology. Such a phenomenon is observed in buoyancy induced motions in the atmosphere, in bodies of water, quasi-solid bodies such as the earth and so on. Many transport processes exist in nature and industrial applications in which the transfer of heat and mass occurs simultaneously as a result of combined buoyancy effects of thermal diffusion and diffusion of chemical species. Some of its engineering applications include the design of heat exchangers, channel type solar energy collectors;

\footnotetext{
* To whom correspondence should be addressed
} 
chemical processes industries such as food processing and polymer production, formation and dispersion of fog, and thermo-protection systems.

Soundalgekar and Warve (1977) analyzed two dimensional unsteady free convection flows past an infinite vertical plate with oscillating wall temperature and constant suction. The effects of suction, which oscillates in time about a constant mean, were also studied by them. An extensive contribution on heat and mass transfer flows was made by Khair and Bejan (1985). Lin and Wu (1995) investigated the problem of simultaneous heat and mass transfer with an entire range of buoyancy ratio for most practical chemical species in dilute and aqueous solutions. Muthucumarswamy et al. (2001) studied the heat and mass transfer effects on flow past an impulsively started infinite vertical plate. The solution was derived using the Laplace transfer techniques, and the effects of the Grashof number, Prandtl number and Schmidt number were discussed.

Stretching surface heat transfer problems have many engineering applications. Materials which are manufactured by extrusion processes and heat treated substances proceeding between a feed roll and a wind-up roll can be classified as continuously stretching surfaces. In order to acquire top-grade property of the final product, the cooling procedure should be effectively controlled. Among related investigations about the stretching surface is the work of Sakiadis (1961) who studied the very basic governing equation on the continuously moving solid surface problem. The flow field of a stretching wall with a power-law velocity variation was discussed by Bank (1983). Ali (1993) extended Bank's work for a power-law stretched surface with suction or injection. Elbashbeshy (1998) also extended the work of Bank for a porous stretched surface with different values of the injection parameter and variable heat flux. Gupta and Gupta (1977) analyzed the stretching problem with constant surface temperature. Sriramula et al. (2001) studied steady flow and heat transfer of a viscous incompressible fluid through a porous medium over a stretching sheet. Elbashbeshy and Bazid $(2003 ; 2004)$ re-analyzed the stretching problem discussed earlier by Elbashbeshy (1998) including variable viscosity, internal heat generation, suction/injection and porous medium. Aboeldahab and Elbarbary (2001) discussed heat and mass transfer along a vertical plate under the combined buoyancy force effects of thermal and species diffusion in the presence of a transversely applied magnetic field and taking Hall effect into account. The system of nonlinear equations was solved by using Runge-Kutta methods.

All the above studies were based on Newtonian viscous fluid flows. Subhas and Veena (1998) investigated a visco-elastic fluid and heat transfer in a porous medium over a stretching surface. Chaudhary and Kumar Jha (2008) investigated the heat and mass transfer in elastico-viscous fluid past an impulsively started infinite vertical plate with Hall effect when the temperature and concentration are assumed to be oscillating with time. They obtained the closed form analytical solutions for velocity, temperature and concentration distributions for various values of the elastic parameter, Schmidt number, magnetic parameters and Hall parameter. Hayat et al. (2008) investigated the hydrodynamic elastico-viscous fluid over a stretching surface. The governing equation of the flow was analytically solved by applying an efficient technique of the homotopy analysis method (HAM). The HAM solution was compared with the known exact solution for the two-dimensional flow and an excellent agreement was found. An analysis was carried out to study the heat transfer characteristics of a second grade non- Newtonian liquid due to a stretching sheet through a porous medium under the influence of external magnetic field by Subhas Abel et al. (2010). They used partial slip condition to study the flow behaviour of the liquid and addressed two types of boundary heating of constant surface temperature and prescribed surface temperature.

Mostafa (2010) examined the study of the boundary layer flow and mass transfer of a visco-elastic fluid immersed in a porous medium over a stretching surface in the presence of surface slip, chemical reaction and variable viscosity. The effects of various involved interesting parameters on the velocity fields and concentration were investigated and discussed. Olajuwon (2011) examined the convection heat and mass transfer in a hydromagnetic flow of a second grade fluid in the presence of thermal radiation and thermal diffusion. He numerically solved the problem using the Runge-Kutta shooting method and presented the velocity, temperature and concentration fields for different values of parameters entering into the problem. 
The present study is an endeavour to investigate the steady mixed convection boundary layer flow of a second grade fluid due to the combined effects of heat and mass transfer over a stretched vertical surface in a porous channel. Numerical solutions have been obtained for the velocity, temperature and species concentration profiles and shown graphically. The results have promising applications in engineering and technology.

\section{Mathematical analysis}

We consider the steady, laminar mixed convective flow of an incompressible second grade fluid with heat and mass transfer due to stretching of a heated or cooled vertical surface of variable temperature $T_{w}(x)$ and variable concentration $C_{w}(x)$ in a porous channel. The fluid is of uniform ambient temperature $T_{\infty}$ and uniform ambient concentration $C_{\infty}$. It is assumed that the surface is stretched in its plane with velocity $u_{w}(x)$. The density variation and buoyancy effects are taken into consideration so that the Boussinesq approximation for both the temperature and species concentration gradient can be adopted. The steady boundary layer equations, in the absence of heat generation and viscous dissipation, are given as follows

$$
\begin{aligned}
& \frac{\partial u}{\partial x}+\frac{\partial v}{\partial y}=0, \\
& u \frac{\partial u}{\partial x}+v \frac{\partial u}{\partial y}=v \frac{\partial^{2} u}{\partial y^{2}}+k_{0}\left(\frac{\partial^{3} u}{\partial x \partial y^{2}}+\frac{\partial u \partial^{2} u}{\partial x \partial y^{2}}+\frac{\partial u \partial^{2} u}{\partial y \partial y^{2}}+v \frac{\partial^{3} u}{\partial y^{3}}\right)+ \\
& -\frac{v}{k} u+g \beta_{T}\left(T-T_{\infty}\right)+g \beta_{C}\left(C-C_{\infty}\right), \\
& u \frac{\partial T}{\partial x}+v \frac{\partial T}{\partial y}=\alpha_{f} \frac{\partial^{2} T}{\partial y^{2}} \\
& u \frac{\partial C}{\partial x}+v \frac{\partial C}{\partial y}=D_{f} \frac{\partial^{2} C}{\partial y^{2}}
\end{aligned}
$$

where $u$ and $v$ represent the velocity components in the $x$ - and $y$-directions, respectively; $T$ is the fluid temperature; $\alpha_{f}$ is the thermal diffusivity; $g$ is the acceleration due to gravity; $\beta_{T}$ is the volumetric coefficient of thermal expansion; $\beta_{C}$ is the volumetric coefficient of concentration expansion; $\rho$ is the density of the fluid; $T_{\infty}$ is the free stream temperature; $C_{\infty}$ is the free stream concentration of the chemical species; $D_{f}$ is the mass diffusivity; $k_{0}$ is the material parameter for second grade fluid.

The boundary conditions at the sheet (wall of the channel) and in the free stream are given by

$$
\begin{aligned}
& u=u_{w}(x)=a x, \quad v=0, \quad T=T_{w}(x)=T_{\infty}+b x, \quad C=C_{w}(x)=C_{\infty}+C x \quad \text { at } \quad y=0 ; \\
& u \rightarrow 0, \quad \frac{\partial u}{\partial y} \rightarrow 0, \quad T \rightarrow T_{\infty}, \quad C \rightarrow C_{\infty} \quad \text { as } \quad y \rightarrow \infty .
\end{aligned}
$$

To facilitate the solution of this problem, we introduce similarity transformations as follows 


$$
\eta=\sqrt{\frac{a}{v}} y, \quad \psi=x \sqrt{a v} f(\eta), \quad \theta(\eta)=\frac{T-T_{\infty}}{T_{w}-T_{\infty}}, \quad \varphi(\eta)=\frac{C-C_{\infty}}{C_{w}-C_{\infty}}
$$

The stream function, $\psi$, satisfies the continuity Eq.(2.1) automatically with

$$
u=\frac{\partial \psi}{\partial y} \quad \text { and } \quad v=-\frac{\partial \psi}{\partial x}
$$

In view of the above non-dimensional quantities, the governing Eqs (2.2) - (2.4) are reduced to the following dimensionless forms

$$
\begin{aligned}
& f^{\prime \prime \prime}+f f^{\prime \prime}-f^{\prime 2}-K\left(f f^{i v}-2 f^{\prime} f^{\prime \prime \prime}+f^{\prime \prime 2}\right)-\gamma f^{\prime}+\lambda(\theta+N \varphi)=0, \\
& \theta^{\prime \prime}+\operatorname{Pr} \theta^{\prime}-\operatorname{Pr} \theta f^{\prime}=0, \\
& \varphi^{\prime \prime}+\operatorname{Pr} \operatorname{Le}\left(f \varphi^{\prime}-\varphi f^{\prime}\right)=0,
\end{aligned}
$$

with boundary conditions

$$
\begin{aligned}
& f(0)=0, \quad f^{\prime}(0)=1, \quad \theta(0)=1, \quad \varphi(0)=1, \\
& f^{\prime}(\infty) \rightarrow 0, \quad f^{\prime \prime}(\infty) \rightarrow 0, \quad \theta(\infty) \rightarrow 0, \quad \varphi(\infty) \rightarrow 0 .
\end{aligned}
$$

In the above equations, the second grade parameter $K(\succ 0)$, the constant dimensionless porosity parameter $\gamma$, the constant dimensionless mixed convection parameter $\lambda$, the constant dimensionless concentration buoyancy ratio parameter $N$, the Prandtl number Pr, the Lewis number Le are

$$
K=\frac{k_{0} a}{v}, \quad \gamma=\frac{v}{a K}, \quad \lambda=\frac{\mathrm{Gr}_{x}}{\operatorname{Re}_{x}^{2}}, \quad N=\frac{\beta_{C}\left(C_{w}-C_{\infty}\right)}{\beta_{T}\left(T_{w}-T_{\infty}\right)}, \quad \operatorname{Pr}=\frac{v}{\alpha_{f}}, \quad \operatorname{Le}=\frac{\alpha_{f}}{D_{f}},
$$

with $\mathrm{Gr}_{x}=\frac{g \beta_{T}\left(T_{w}-T_{\infty}\right) x^{3}}{v^{2}}$ being the local Grashof number and $\operatorname{Re}_{x}=\frac{U_{w} x}{v}$ is the local Reynolds number.

\section{Numerical procedure}

The set of non-linear ordinary differential Eqs (2.8)-(2.10) with the boundary conditions in Eq.(2.11) was solved by using the Runge-Kutta Fehlberg integration scheme along with shooting iteration technique with $K, \gamma, \lambda, N, \operatorname{Pr}$, and Le as prescribed parameters. A step-size of $\Delta \eta=0.001$ is selected to be satisfactory for a convergence criterion of $10^{-5}$ in nearly all cases. The value of $y_{\infty}$ is found for each iteration loop by the assignment statement $\eta_{\infty}=\eta_{\infty}+\Delta \eta$. The maximum value of $\eta_{\infty}$, to each group of parameters $K$, $\gamma, \lambda, N, \operatorname{Pr}$, Le is determined when the values of the unknown boundary conditions at $\eta_{\infty}=0$ do not change to successfully loop with an error less than $10^{-5}$. 


\section{Results and discussion}

For discussing the results, the numerical calculations are presented in the form of the nondimensional velocity, temperature and species concentration profiles. In the computations, the physical realistics numerical values are assigned to the embedded parameters in the system of coupled-equations with respect to the profiles. The variation of $f^{\prime}, \theta$ and $\varphi$ versus $\eta$ is desired for the velocity, temperature and species concentration boundary layer, respectively. The values of skin-friction coefficient, the local Nussselt number and the local Sherwood number for various values of embedded parameters are shown in Tab.1. It is obvious that $\lambda \succ 0, \lambda \prec 0$ and $\lambda=0$ correspond to assisting flow (heated plate), opposing flow (cooled plate) and forced convection flow, respectively. It must also be noted that $N$ can take positive values or negative values and $N$ can be zero (absence of mass transfer). It is further observed that an increase in the porosity parameter $\gamma$ and Prandtl number Pr leads to an increase in the skin-friction at the wall surface. However, an increase in the second grade parameter $K$, bouyancy ratio parameter $N$, mixed convection parameter $\lambda$ and Lewis number Le decreases the skin-friction at the stretching wall surface. Similarly, the Nusselt number increases at the stretching wall surface when the porosity parameter $\gamma$, mixed convection parameter $\lambda$, Prandtl number $\operatorname{Pr}$, bouyancy ratio parameter $N$ and Lewis number Le increase while it decreases at the wall surface when the second grade parameter $K$ increases. It is interesting to notice that increasing the second grade parameter $K$, mixed convection parameter $\lambda$, Prandtl number Pr, bouyancy ratio parameter $N$ and Lewis number Le leads to an increase in the Sherwood number Sh (i.e., the concentration boundary layer thickness). Also, an increase in the porosity parameter $\gamma$ resulted in shear thinning of the concentration boundary layer thickness (increase in the Sherwood number). Finally, it is observed that the flow field is appreciably influenced by the porosity and second grade parameters.

Table 1. Numerical computations showing $-f^{\prime \prime}(0),-\theta^{\prime}(0)$ and $-\varphi^{\prime}(0)$.

\begin{tabular}{|l|l|l|l|l|l|l|l|l|}
\hline$K$ & $\gamma$ & $\lambda$ & $N$ & $\operatorname{Pr}$ & Le & $-f^{\prime \prime}(0)$ & $-\theta^{\prime}(0)$ & $-\varphi^{\prime}(0)$ \\
\hline 0.1 & 0.1 & 1 & -0.2 & 1 & 1 & 0.72050216693 & 1.4681813314 & 1.0571489966 \\
\hline 0.2 & 0.1 & 1 & -0.2 & 1 & 1 & 0.70451645128 & 1.4717584190 & 1.0626470072 \\
\hline 0.4 & 0.1 & 1 & -0.2 & 1 & 1 & 0.67458386298 & 1.4782689644 & 1.0724906790 \\
\hline 1.0 & 0.1 & 1 & -0.2 & 1 & 1 & 0.60173391780 & 1.4936773446 & 1.0950158732 \\
\hline 0.2 & 0.2 & 1 & -0.2 & 1 & 1 & 0.74795240003 & 1.4652357579 & 1.0524928075 \\
\hline 0.2 & 0.4 & 1 & -0.2 & 1 & 1 & 0.83045712096 & 1.4530701699 & 1.0330878370 \\
\hline 0.2 & 0.1 & 2 & -0.2 & 1 & 1 & 0.47377024190 & 1.4977728687 & 1.1022598741 \\
\hline 0.2 & 0.1 & 3 & -0.2 & 1 & 1 & 0.25905130774 & 1.5199236365 & 1.1348984132 \\
\hline 0.2 & 0.1 & 1 & -0.3 & 1 & 1 & 0.74006769447 & 1.4672257468 & 1.0555659033 \\
\hline 0.2 & 0.1 & 1 & -0.4 & 1 & 1 & 0.77671104469 & 1.4623829485 & 1.0479365482 \\
\hline 0.2 & 0.1 & 1 & -0.2 & 3.0 & 1 & 0.85221694823 & 3.6714839197 & 1.9481935653 \\
\hline 0.2 & 0.1 & 1 & -0.2 & 7.1 & 1 & 0.99288005780 & 7.9014275557 & 2.9439130470 \\
\hline 0.2 & 0.1 & 1 & -0.2 & 1 & 2 & 0.68514221210 & 1.4758096755 & 1.5940019673 \\
\hline 0.2 & 0.1 & 1 & -0.2 & 1 & 5 & 0.66808427050 & 1.4782678643 & 2.6340761676 \\
\hline 0.2 & 0.1 & 1 & -0.2 & 1 & 4 & 0.65920959575 & 1.4791376443 & 3.7998978563 \\
\hline
\end{tabular}




\subsection{Velocity profiles}

The effect of various thermophysical parameters on the non-dimensional fluid velocity profiles are illustrated in Fig.1 to Fig.5. Figure 1 shows the effect of the second grade parameter $K$ on the fluid velocity profiles. It is observed that an increase in the dimensionless second grade parameters $K$ leads to an increase in the fluid velocity thereby thickening the velocity boundary layer. Figure 2 captures the influence of increasing the porosity parameter $\gamma$, this decreases the fluid velocity which leads to the velocity boundary layer thinning. Also, Fig. 3 clearly depicts that the mixed convection parameter $\lambda \succ 0$ corresponds to assisting flow (heated plate). An increase in the mixed convection parameter $\lambda$ results in an increase in the fluid velocity. In Fig.4, velocity profiles across the boundary layer for different values of the Prandtl number Pr are illustrated. It is evident that an increase in the Prandtl number Pr corresponds to a decrease in the fluid velocity. Figure 5 displays the velocity profiles for various values of the dimensionless buoyancy ratio parameters $N . N \prec 0$ slows down the fluid velocity whereas $N \succ 0$ increase the fluid velocity.

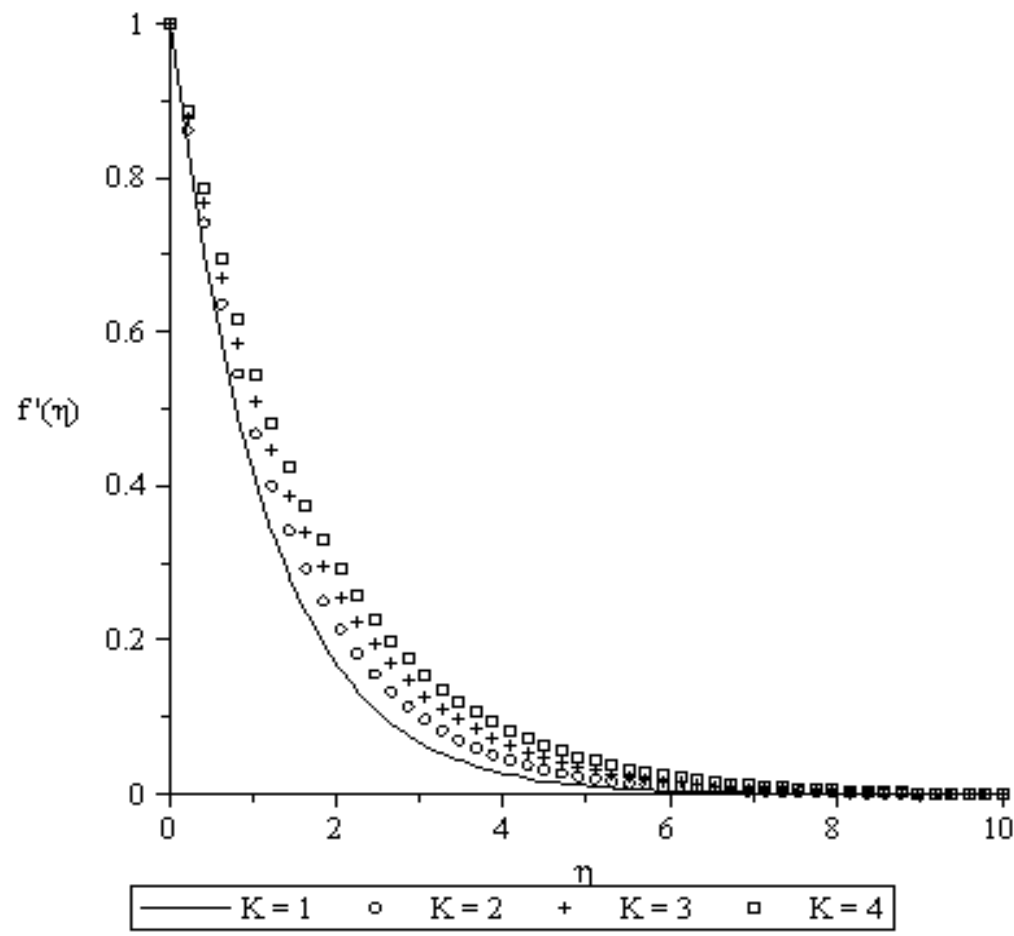

Fig.1. Effects of the second grade parameter $K$ on the velocity profile when $\operatorname{Pr}=1, N=-0.2, \mathrm{Le}=1, \lambda=1$, $\gamma=1$. 


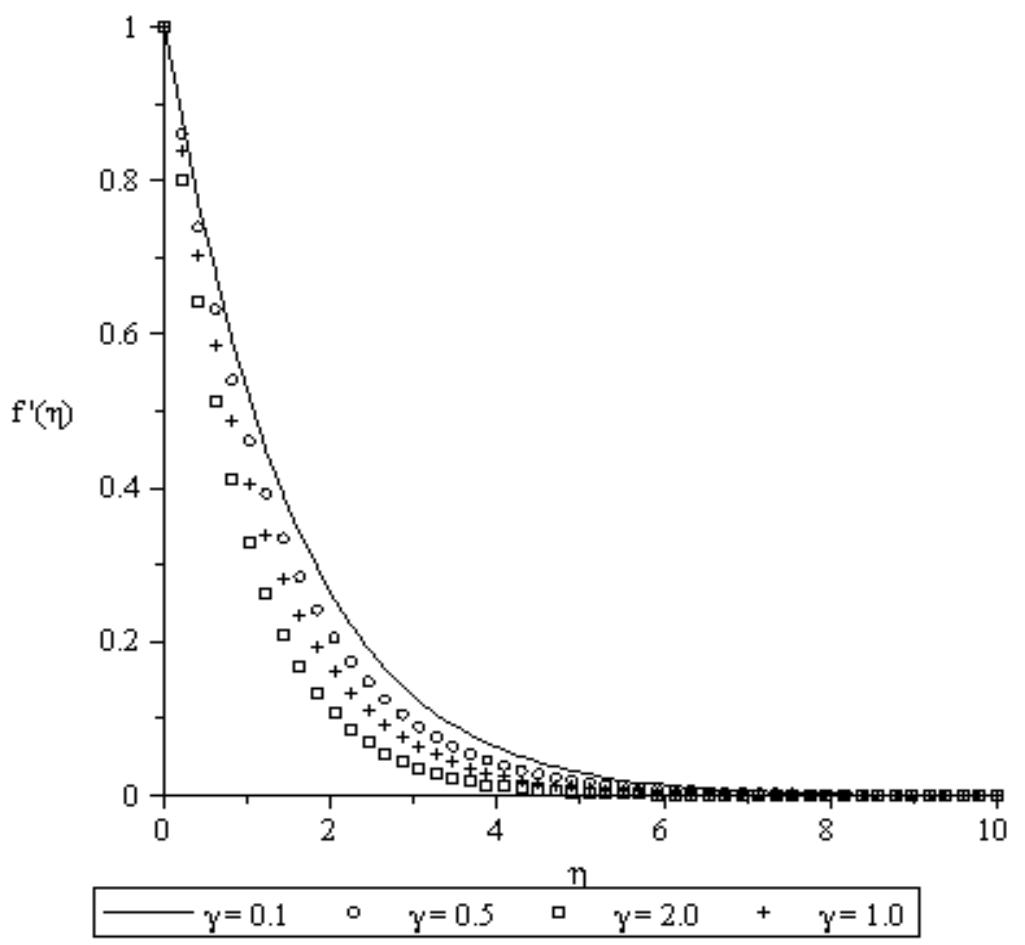

Fig.2. Effects of the porosity parameter $\gamma$ on the velocity profile when $\operatorname{Pr}=1, N=-0.2, \mathrm{Le}=1, \lambda=1, K=1$.

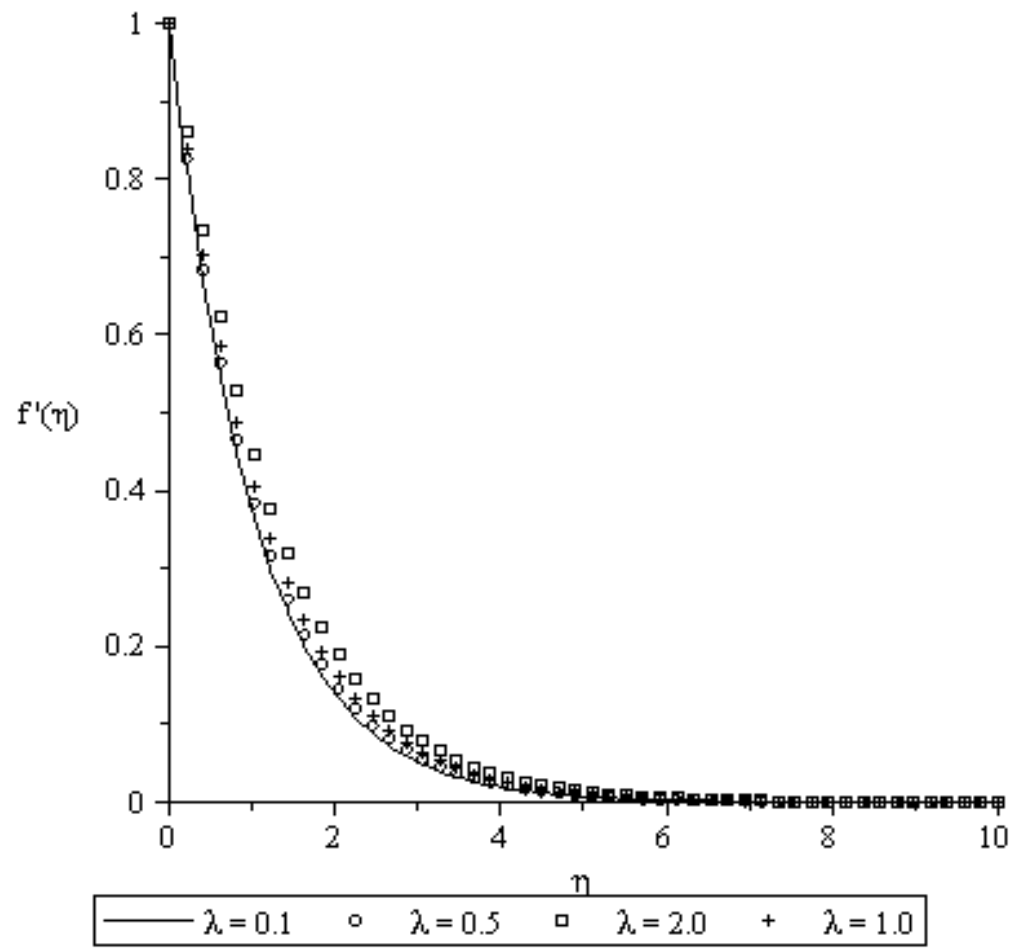

Fig.3. Effects of the mixed convection parameter $\lambda$ on the velocity profile when $\operatorname{Pr}=1, N=-0.2, \operatorname{Le}=1, \gamma=1$, $K=1$. 


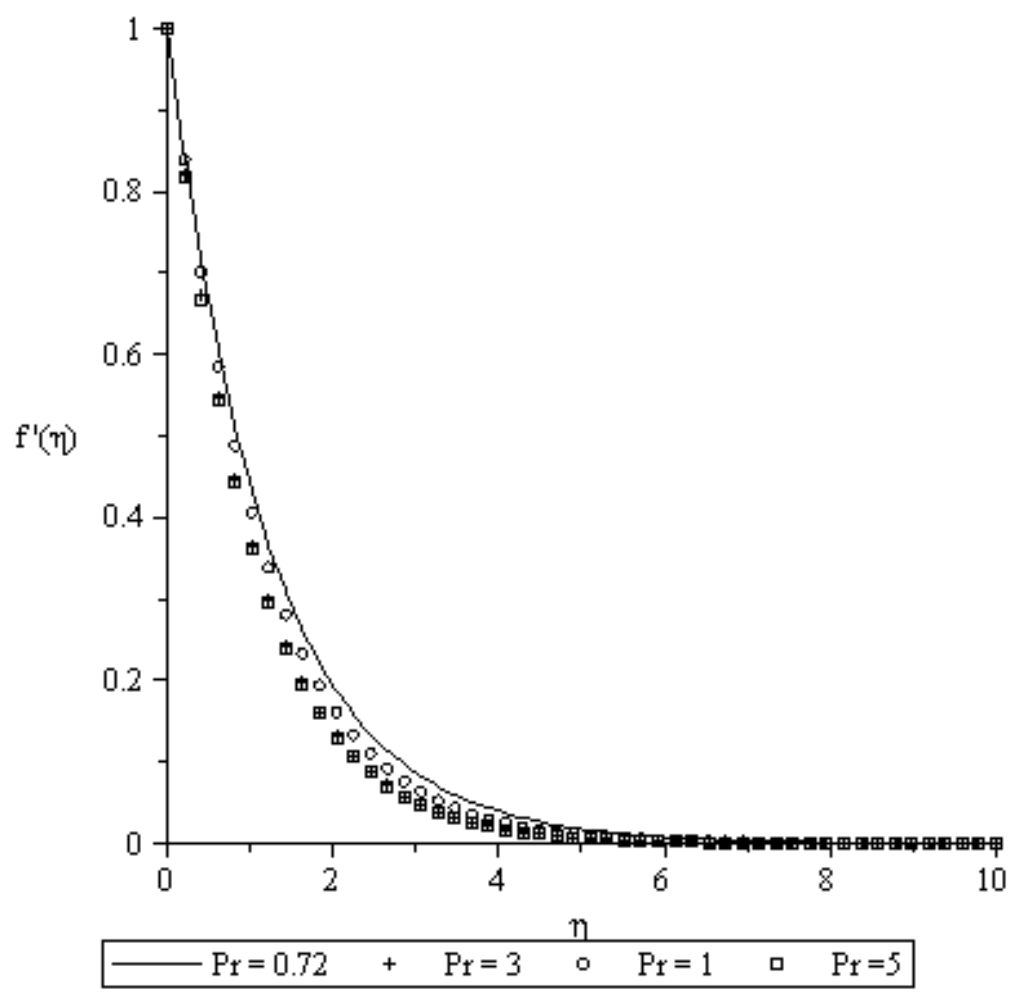

Fig.4. Effects of the Prandtl number Pr on the velocity profile distribution when $\lambda=1, N=-0.2, \mathrm{Le}=1, \gamma=1$, $K=1$.

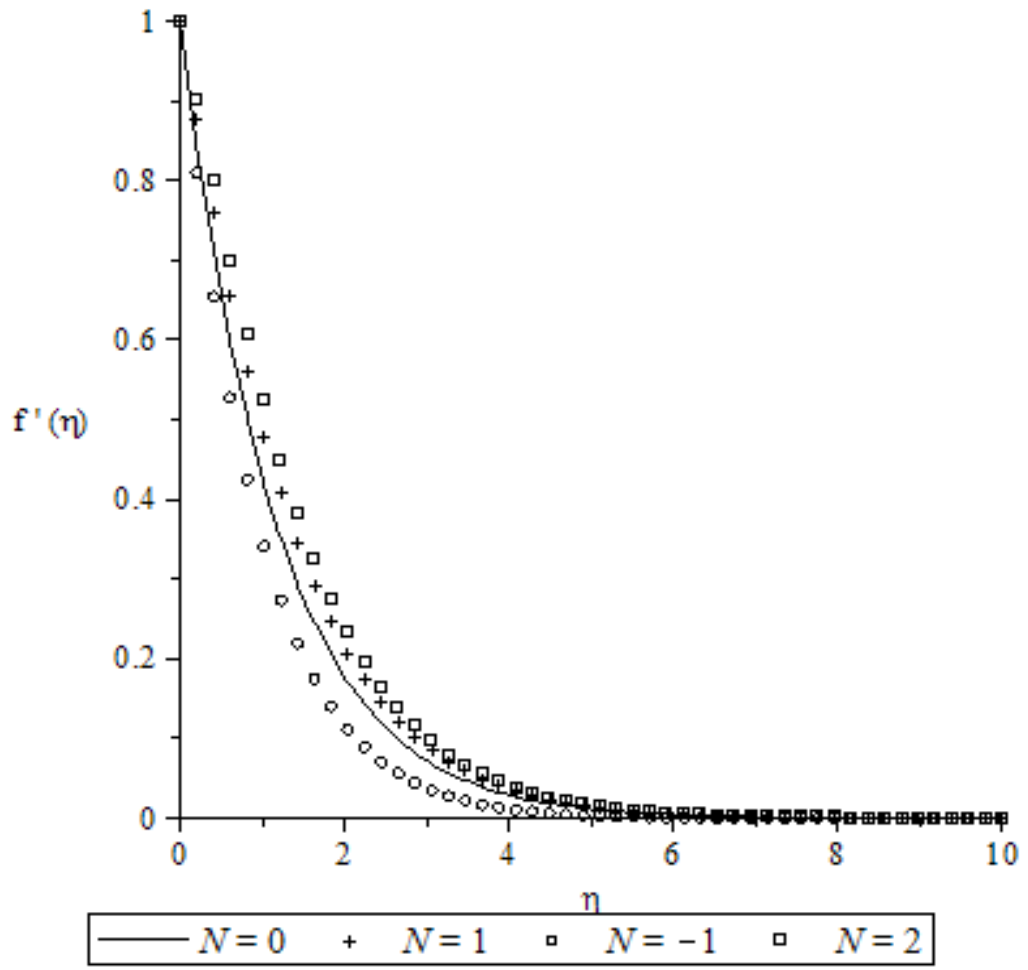

Fig.5. Effects of the mixed convection parameter $N$ on the velocity profile when $\lambda=1, \operatorname{Pr}=1, \mathrm{Le}=1$, $\gamma=1, K=1$. 


\subsection{Temperature profiles}

Figures 6-10 express the influences of the embedded parameters in the flow on non-dimensional temperature profiles. Figure 6 shows the variations of the Prandtl number Pr on the temperature distribution. The thermal boundary layer thickness if found to decrease upon increasing the Prandtl number Pr. This is expected because larger Prandtl numbers correspond to the weaker thermal diffusivity and thinner boundary layer. In Figs 7, 8 and 9, it is observed that the variation of the second grade parameters $K$, porosity parameter $\gamma$ and mixed convection parameter $\lambda$ has little effect on the fluid temperature. It is interesting to note that in Fig.7, the temperature profiles for values of the dimensionless second grade parameter $K$ reveal that increase in the second grade parameter $K$ resulted in a slight decrease in the fluid temperature. Figure 8 illustrates the temperature profiles across the boundary layer thickness for different values of the non-dimensional porosity parameter $\gamma$ and is noticed that increasing the porosity parameters $\gamma$ leads to a small increase in the fluid temperature. Figure 9 also displays the effect for different values of the dimensionless mixed convection parameter $N$ on the temperature profiles. It is observed that an increase in the mixed convection parameter $N$ gives a very slight decrease in the fluid temperature. Figure 10 the effects of the buoyancy ratio parameter $\mathrm{N}$ on the temperature profile. An increase in the buoyancy ratio parameter $N$ decreases the dimensionless temperature distribution.

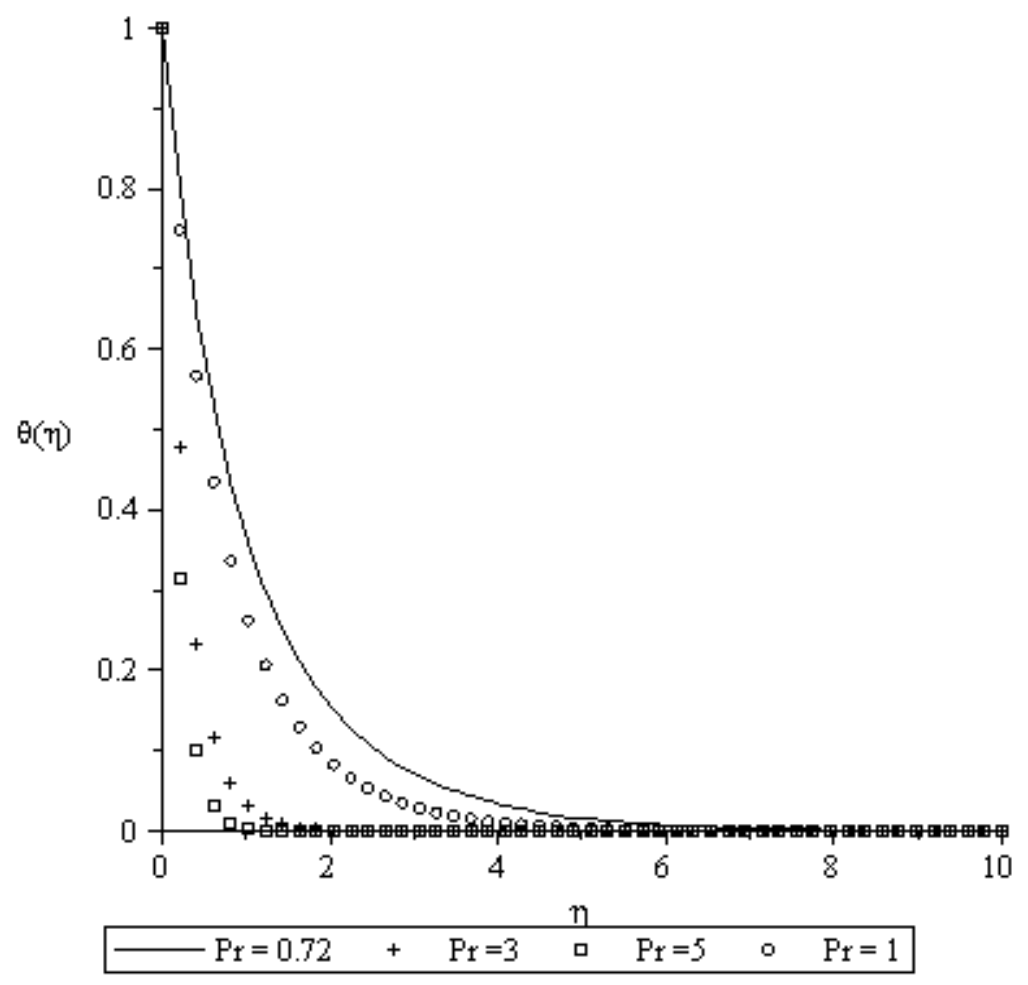

Fig.6. Effects of the Prandtl number Pr on the temperature profile when $\lambda=1, N=-0.2$, Le $=1, \gamma=1, K=1$. 


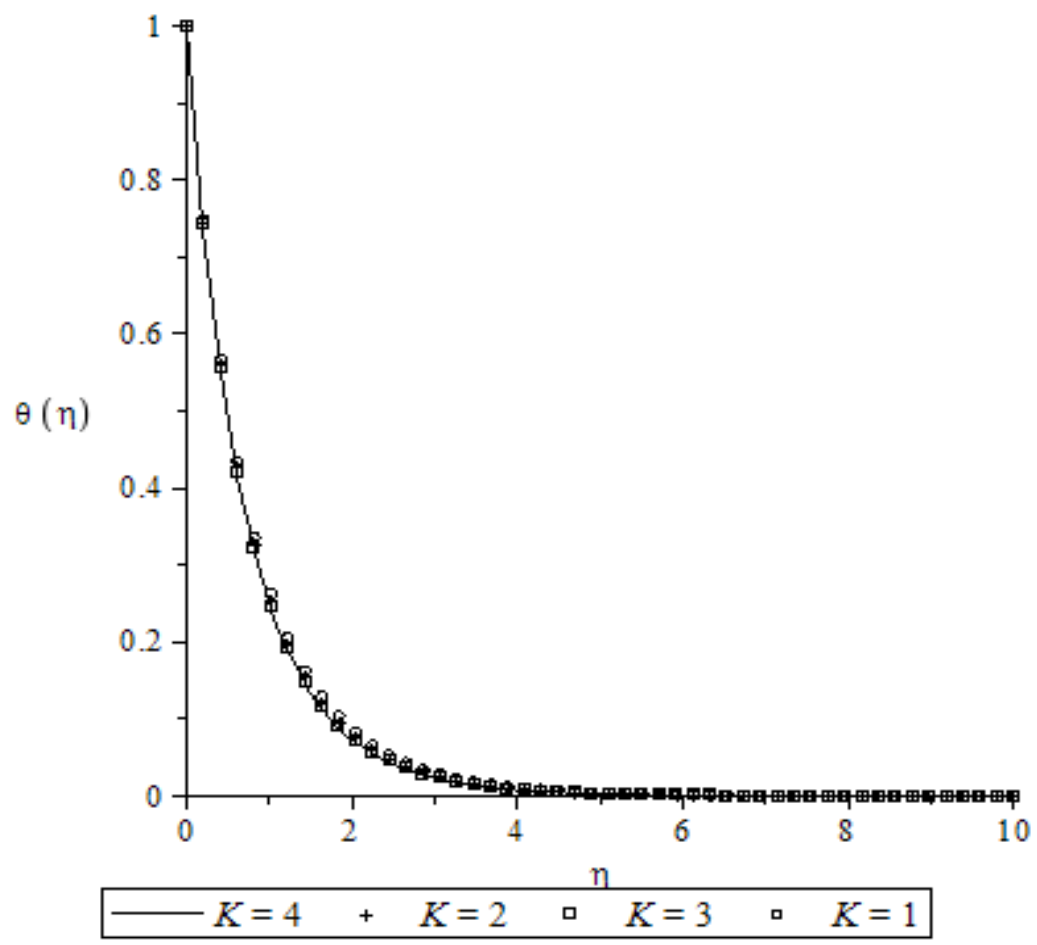

Fig.7. Effects of the second grade parameter $K$ on the temperature profile when $\operatorname{Pr}=1, N=-0.2, \operatorname{Le}=1, \lambda=1$, $\gamma=1$.

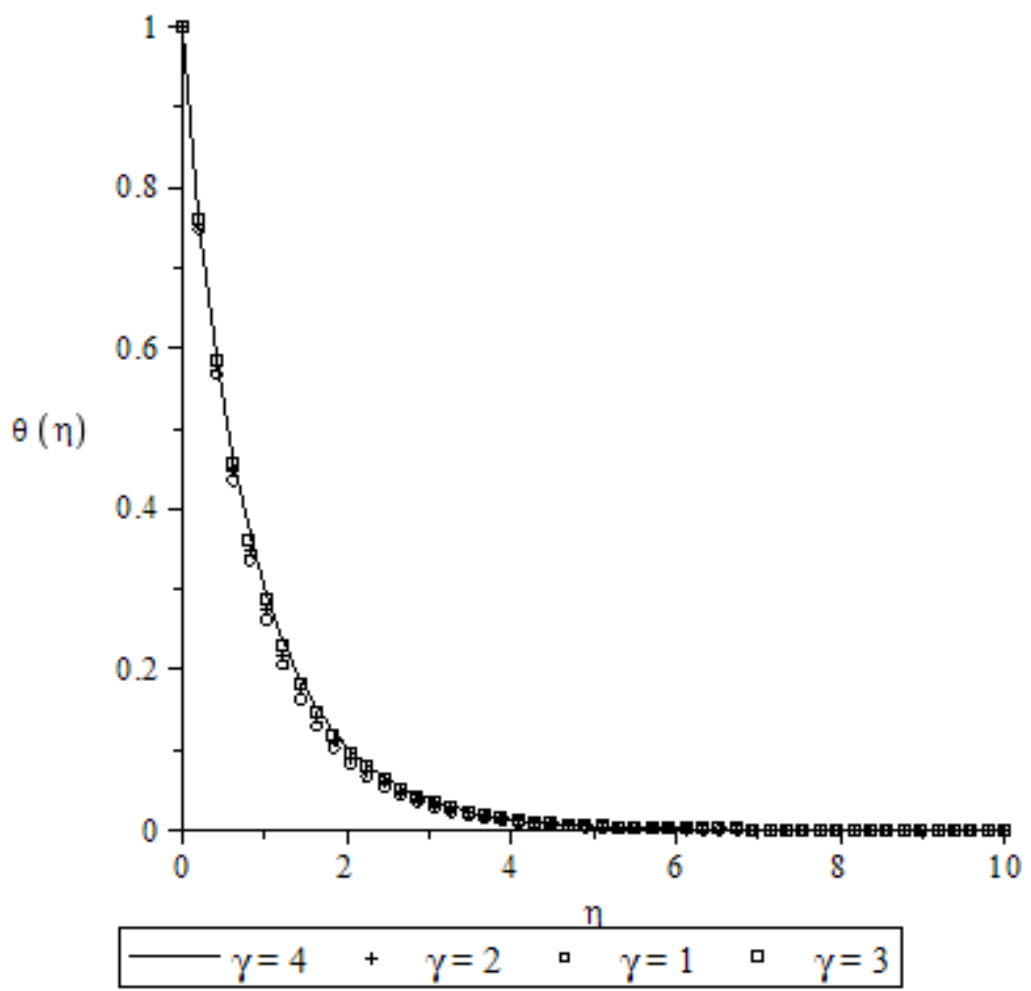

Fig.8. Effects of the porosity parameter $\gamma$ on the temperarure profile when $\operatorname{Pr}=1, N=-0.2, \mathrm{Le}=1, \lambda=1, K=1$. 


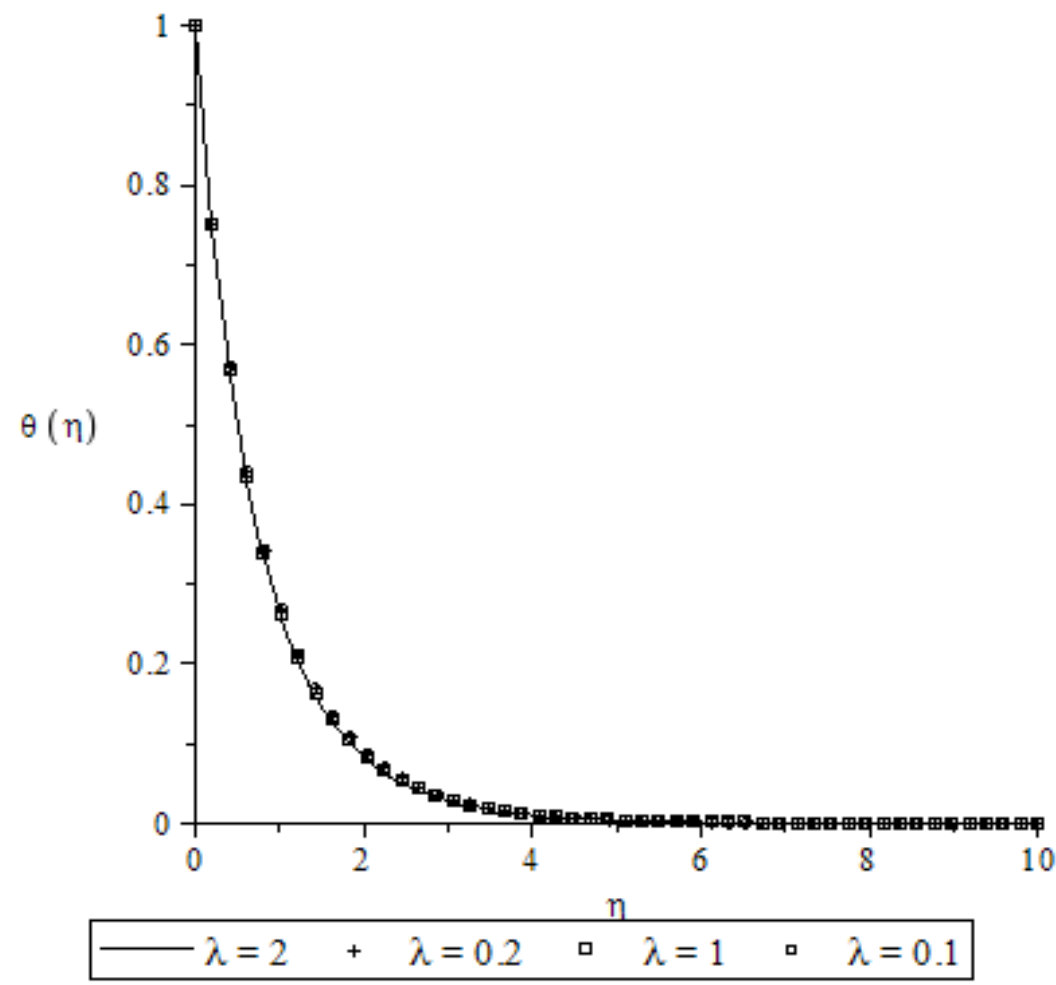

Fig.9. Effects of the mixed convection parameter $\lambda$ on the temperature distribution when $\operatorname{Pr}=1, N=-0.2$, $\mathrm{Le}=1, \gamma=1, K=1$.

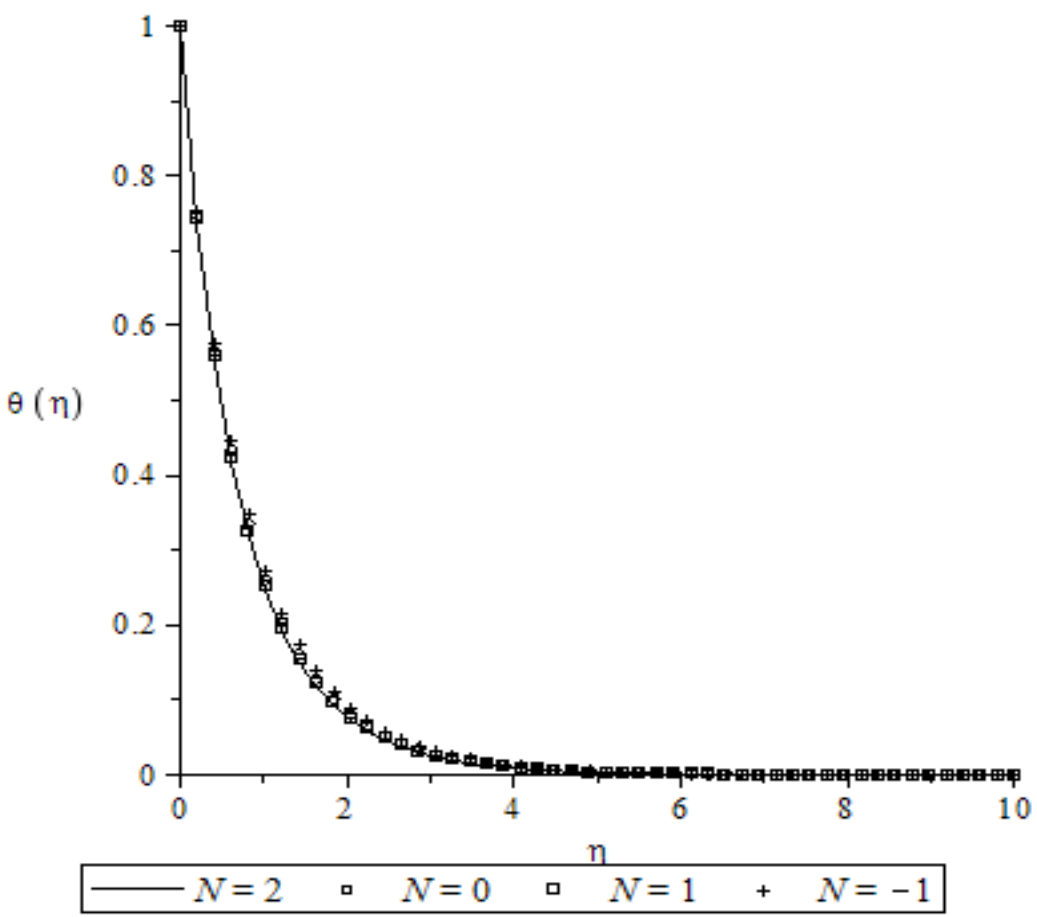

Fig.10. Effects of the buoyancy ratio parameter $N$ on the temperature distribution when $\operatorname{Pr}=1, N=-0.2$, Le $=1, \gamma=1, K=1$. 


\subsection{Concentration profiles}

The influences of the embedded parameters on the concentration profiles are displayed in Figs 11 to 16. Figures 11 and 12 display the variations of the second grade parameter $K$ and porosity parameter $\gamma$ on the species concentration profiles. It is obvious that an increase in the second grade parameters $K$ decreases the concentration profiles while an increase in the porosity parameter increases the species concentration field. The effects of these parameters are similar to that of the temperature field. However, these consequences are more pronounced in the species concentration field than the temperature field when Figs: 7 versus 11,8 versus 12,9 versus 13 and 10 versus 16 are compared. The influences of the mixed convection parameters $\lambda$ and Prandtl number Pr on the species concentration profiles are similar as shown by Figs 13 and 14. When the mixed convection parameter $\lambda$ and Prandtl number Pr increase, the species concentration boundary layer thickness increases. Figure 15 displays the species concentration profile for various values of the Lewis number Le. The effect of Lewis numbers on the concentration profile is as follows: the Lewis number Le increases gradually, this corresponds to the weaker molecular diffusivity and thinner concentration boundary layer thickness. Finally, in Fig.16, the concentration field for different values of the dimensionless species concentration buoyancy parameter $N$ is displayed. It is observed that when the buoyancy parameters $N \succ 0$, the species concentration boundary layer thickness decreases and increases when the buoyancy parameter $N \prec 0$ as in the case of the temperature field.

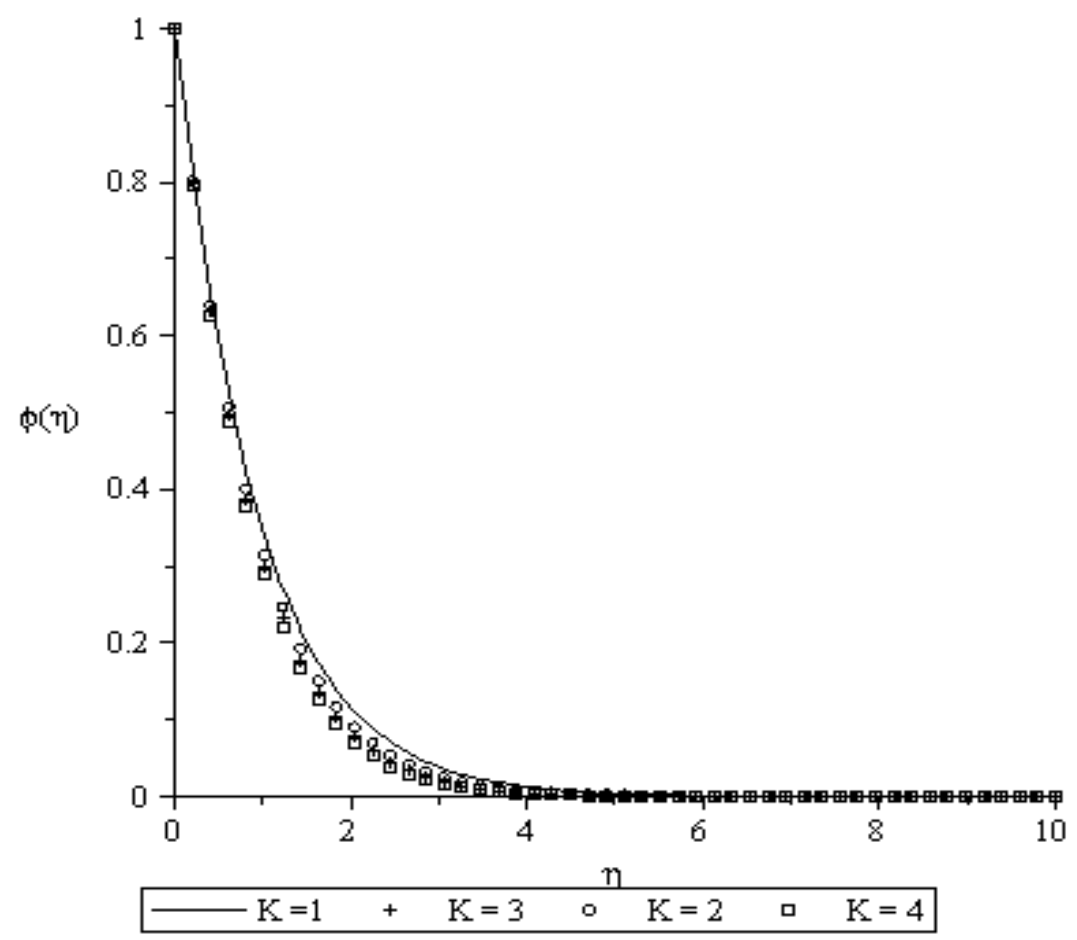

Fig.11. Effects of the second grade parameter $K$ on the concentration distribution when $\operatorname{Pr}=1, N=-0.2$, Le $=1, \lambda=1, \gamma=1$. 


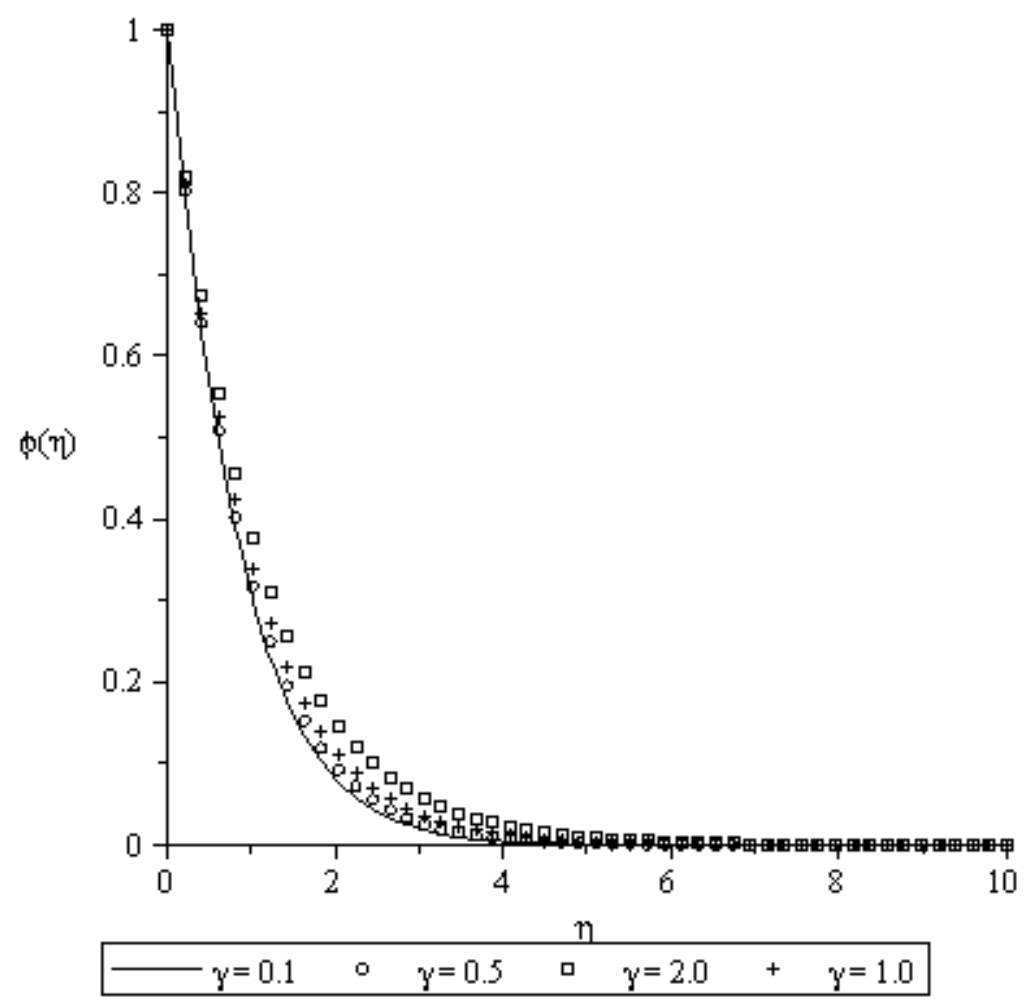

Fig.12. Effects of the porosity parameter $\gamma$ on the concentration distribution when $\operatorname{Pr}=1, N=-0.2, \operatorname{Le}=1$, $\lambda=1, K=1$.

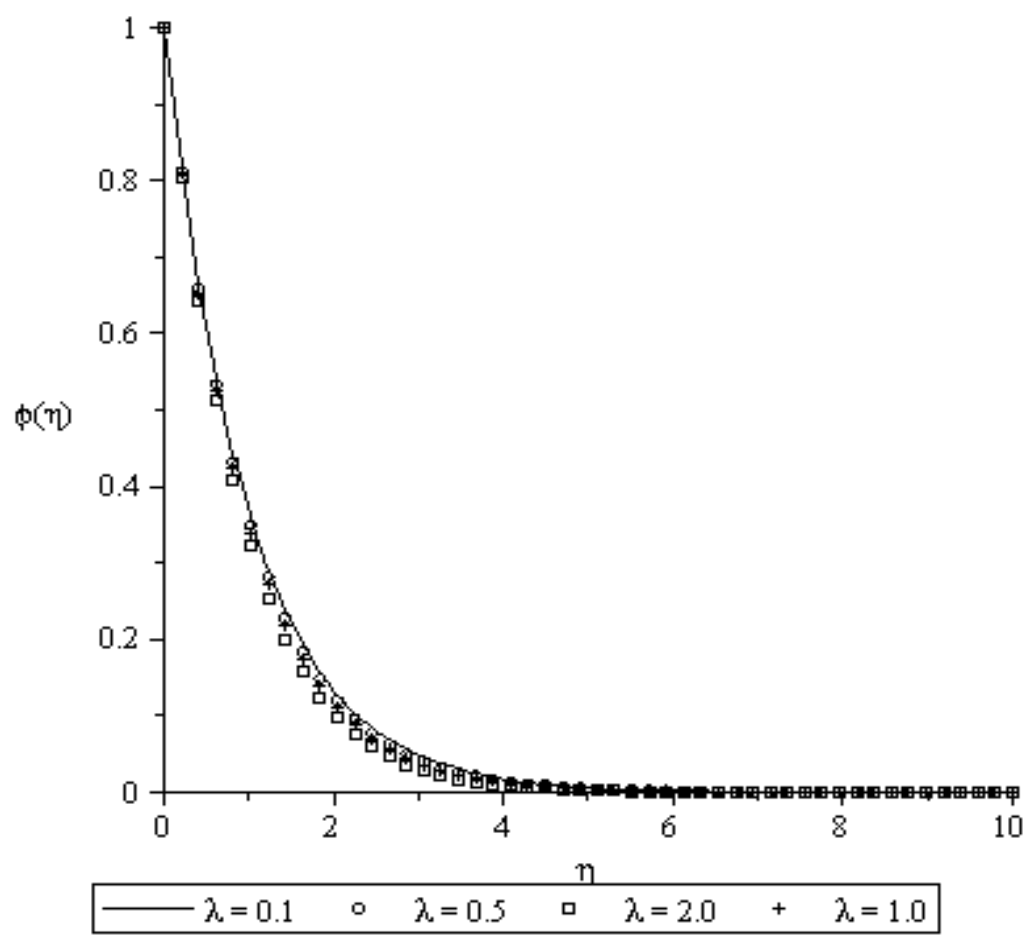

Fig.13. Effects of the mixed convection parameter $\lambda$ on the concentration distribution when $\operatorname{Pr}=1, N=-0.2$, $\mathrm{Le}=1, \gamma=1, K=1$. 


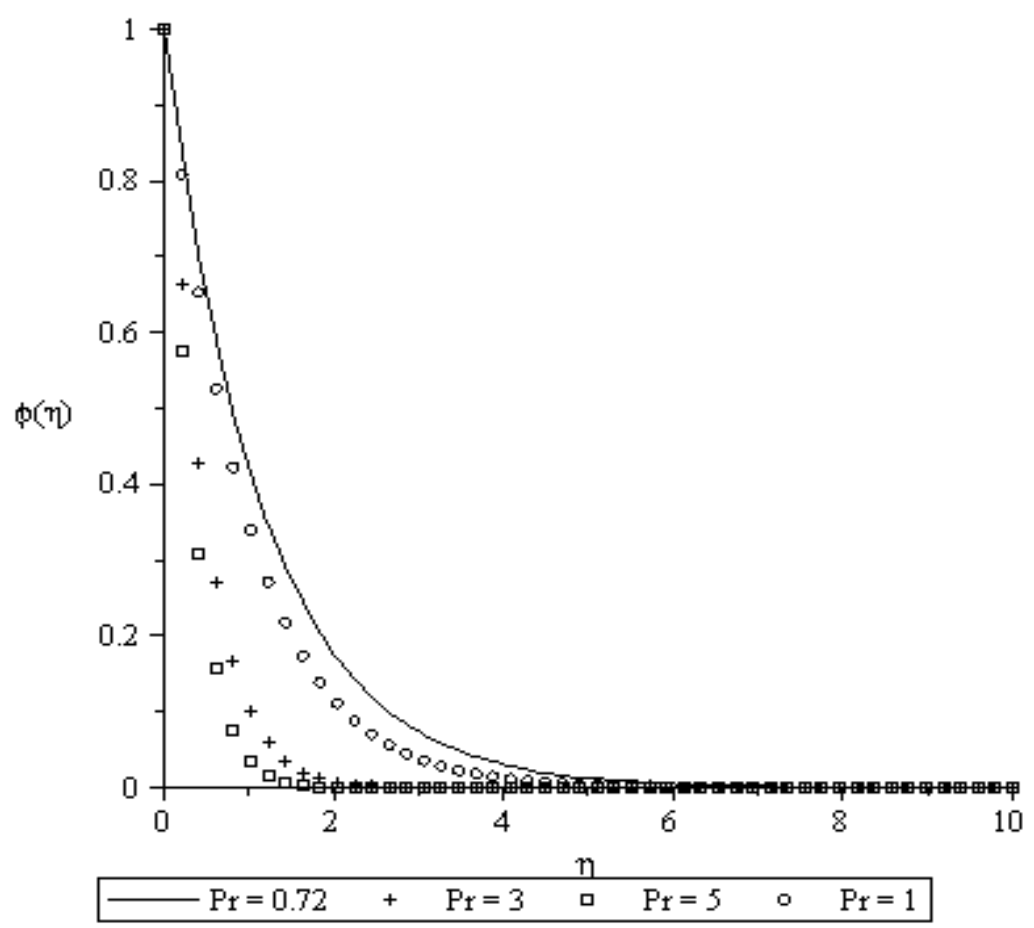

Fig.14. Effects of the Prandtl number Pr on the concentration distribution when $\lambda=1, N=-0.2, \mathrm{Le}=1, \gamma=1$, $K=1$.

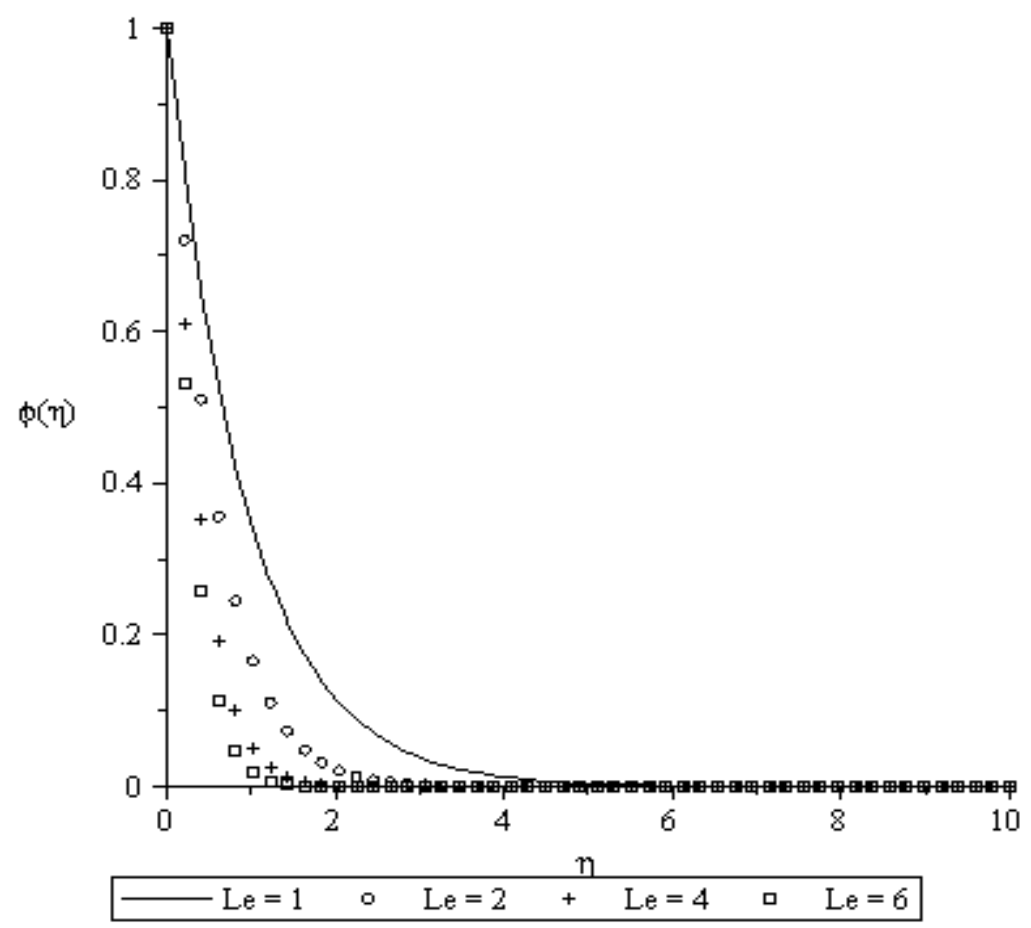

Fig.15. Effects of the Lewis number Le on the concentration distribution when $\lambda=1, N=-0.2, \operatorname{Pr}=1, \gamma=1$, $K=1$. 


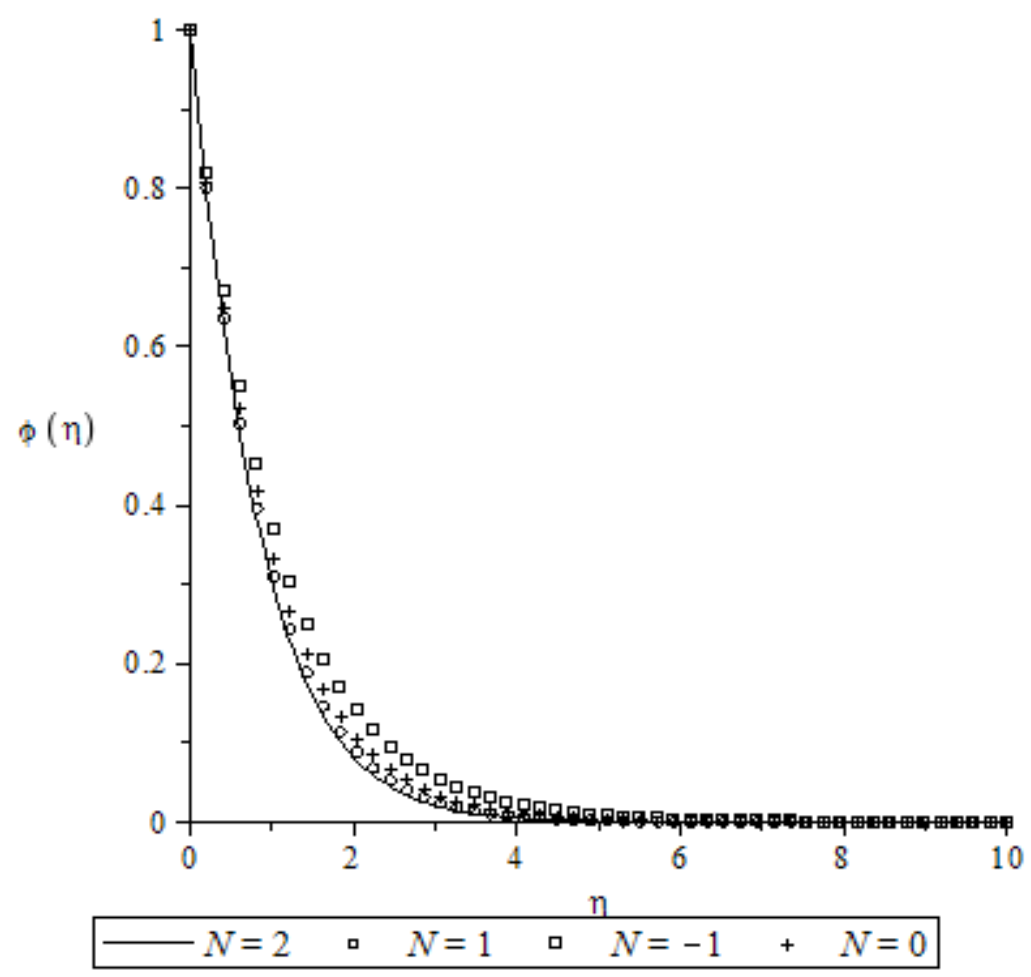

Fig.16. Effects of the mixed convection parameter $N$ on the concentration distribution when $\lambda=1, \operatorname{Pr}=1$, $\mathrm{Le}=1, \gamma=1, K=1$.

\section{Conclusion}

A simultaneous heat and mass transfer in a mixed convection boundary layer flow of a second grade fluid over a stretching vertical surface in a porous channel is examined. The partial differential equations governing the problem have been transformed by a similarity transformation into a system of coupled ordinary differential equations which are solved numerically by using the shooting method with the fifthorder Runge-Kutta technique. Dimensionless velocity, temperature and species concentration profiles are graphically displayed for different values of parameters embedded in the flow model. Our results show among others that:

- The boundary layer fluid velocity increases as the second grade parameter $K$, mixed convection parameter $\lambda$ and Prandtl number Pr increase. Also, the concentration buoyancy ratio parameter $N \succ 0$ increases the fluid velocity and $N \prec 0$ slows down the fluid velocity;

- The thermal boundary layer thickness decreases slightly with an increase in the second grade parameter $K$, Prandtl number Pr, buoyancy ratio parameter $N$ and mixed convection parameter and it increases slightly as the porosity parameters $\gamma$ increases;

- The species concentration boundary layer thickness increases with a decrease in the second grade parameter $K$, Prandtl number Pr, mixed convection parameter $\lambda$, Lewis number Le and concentration buoyancy parameter $N$ and it increases as the porosity parameter $\gamma$ increases;

- The local skin-friction at the plate increases with an increase in the porosity parameter $\gamma$ and Prandtl number Pr and it decreases with an increase in the second grade $K$, and mixed convection parameter $\lambda$, buoyancy ratio parameter $N$ and Lewis number Le; 
- The rate of heat transfer at the plate increases as the porosity parameter $\gamma$, mixed convection parameter $\lambda$, Prandtl number Pr and Lewis number Le increase while the second grade parameter $K$ and concentration buoyancy ratio parameter increase;

- The rate of mass transfer at the plate increases as the second grade parameter $K$, Lewis number Le, mixed convection parameter $\lambda$ and Prandtl number Pr increase and it decreases as the porosity parameter $\gamma$ and concentration buoyancy ratio parameter increase.

\section{Nomenclature}

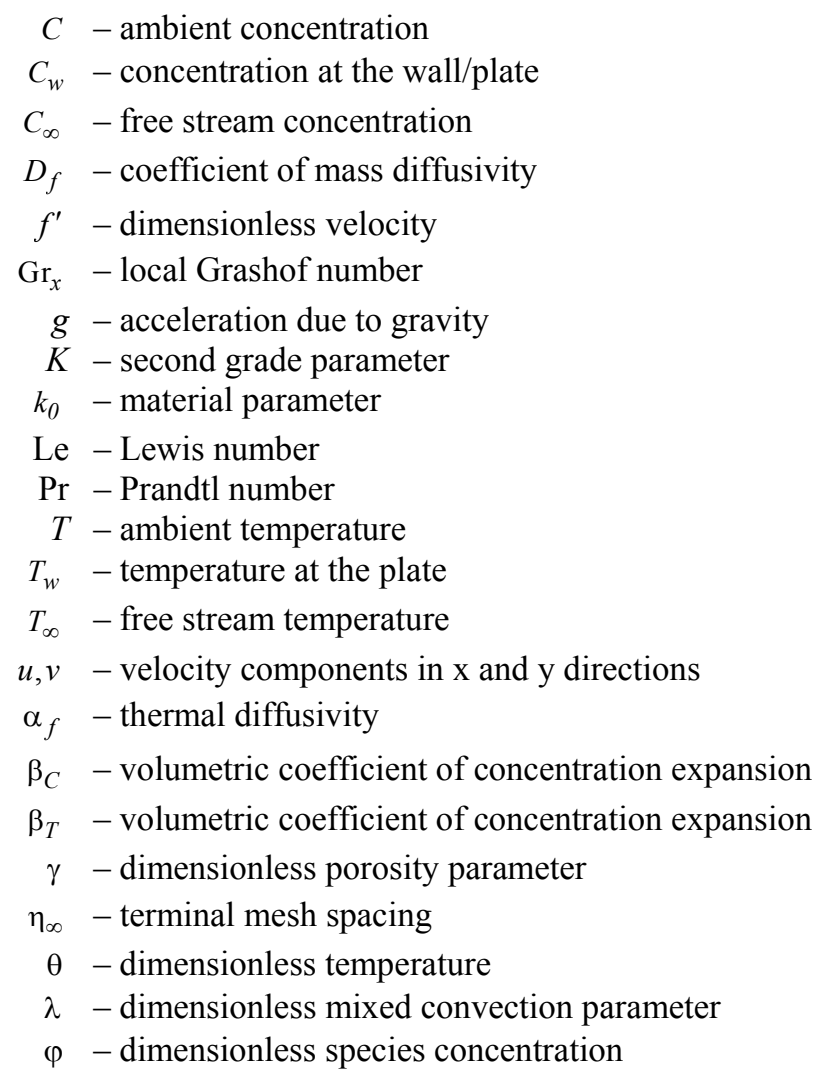

\section{Reference}

Aboeldahab E.M. and Elbarbary E.M.E. (2001): Hall current effect magnetohydrodynamicfree convection flow past a semi-infinite vertical plate with mass transfer. - International Journal of Engineering Sciences, vol.39, pp.16411652.

Ali M.E. (1995): On thermal boundary layer on a power-law stretched surface with suction or injection. - International Journal of Heat Mass Flow, vol.16, pp.280-290.

Banks W.H.H. (1983): Similarity solutions of the boundary layer equation for a stretching wall. - Journal of Mechanical Theory and Applications, vol.2, pp.375-392.

Chaudhary R.C. and Kumar Jha A. (2008): Heat and mass transfer in elastico-viscous fluid past an impulsively started infinite vertical plate with Hall effect. - Latin American Applied Research, vol.38, pp.17-26.

Elbashbeshy E.M.A. (1998): Heat transfer over a stretching surface with variable heat flux. - Journal of Physics D: Appl. Physics, vol.31, pp.1951-1955.

Elbashbeshy E.M.A. and Bazid M.A.A. (2003): Heat transfer over a stretching surface with internal heat generation. Canadian Journal of Physics, vol.81, No.4, pp.699-703. 
Elbashbeshy E.M.A. and Bazid M.A.A. (2004): Heat transfer in a porous medium over a stretching surface with internal heat generation and suction or injection. - Applied Mathematics and Computation, vol.158, No.3, pp.799807.

Gupta P.S. and Gupta P.A. (1977): Heat and mass transfer on a stretching sheet with suction or blowing. - Canadian Journal of Chemical Engineering, vol.55, No.6, pp.744-746.

Hayat T., Sajid M. and Pop I. (2008): Three-dimensional flow over a stretching surface in a visco-elastic fluid. Nonlinear Analysis: Real World Applications, vol.9, No.4, pp.1811-1822.

Khair K.R. and Bejan A. (1985): Mass transfer to natural convection boundary layer flow driven by heat transfer. Journal of Heat Transfer, vol.107, pp.979-981.

Lin H.T. and Wu C.M. (1995): Combined heat and mass transfer by laminar natural convection from a vertical plate. Journal of Heat and Mass Transfer, vol.30, pp.369-376.

Mostafa A.A. Mahmoud (2010): Chemical reaction and variable viscosity effects on flow and mass transfer of a nonNewtoman visco-elastic fluid past a stretching surface embedded in a porous medium. - Meccanica, vol.45, No.6, pp.835-846.

Muthucumaraswamy R., Ganeshan P. and Soundalgekar V.M. (2001): Heat and mass transfer effects on flow past on impulsively started vertical plate. - Acta Mechanica, vol.146, pp.1-8.

Olajuwon B.I. (2011): Convection heat and mass transfer in a hydromagnetic flow of a second grade fluid in the presence of thermal radiation and thermal diffusion. - International Communications in Heat and Mass Transfer, vol.38, No.3, pp.377-382.

Sakiadis B.C. (1961): Boundary layer behaviour on continuous solid surface: I Boundary layer on a continuous flat surface. - AlChE Journal 7, vol.2, pp.213-215.

Soundalgekar V.M. and Warve P.D. (1977): Unsteady free convection flow past an infinite vertical plate with mass transfer. - International Journal of Heat and Mass Transfer, vol.20, pp.1363-1373.

Subhas Abel M., Mahesha N. and Sharanagouda B. Malipatil (2010): Heat transfer due to MHD slip flow of a second grade liquid over a stretching sheet through a porous medium with non-uniform heat source/sink. - Chemical Engineering Communications, vol.198, No.2, pp.191-213.

Suhas A. and Veena P. (1998): Visco-elastic fluid flow and heat transfer in porous medium over a stretching sheet. International Journal of Non-Linear Mechanics, vol.33, No.3, pp.531-540.

Sriramalu A., Kishan N. and Anand R.J. (2001): Steady flow and heat transfer of a viscous incompressible fluid flow through porous medium over a stretching sheet. - Journal of Energy, Heat and Mass Transfer, vol.23, pp.483495. 